\title{
Application de la vidéo pour le suivi de l'impact des travaux de défense du littoral Valras-Plage (Hérault)
}

\author{
Pierre GAUFRES ${ }^{1}$, Bruno ANDRES ${ }^{1}$, Alexandre TROUVILLIEZ ${ }^{1}$, \\ Didier RIHOUEY ${ }^{2}$, Jérémy DUGOR ${ }^{2}$
}

\author{
${ }^{1}$ CETMEF (Centre d'Etudes Techniques Maritimes Et Fluviales) \\ 2, Bd Président Kennedy, CS 90385, 13097 Aix-en-Provence cedex 2 \\ pierre.gaufres@developpement.durable.gouv.fr, \\ bruno.andres@developpement-durable.gouv.fr, \\ ${ }^{2}$ CASAGEC - Université de Pau et des pays de l'Adour \\ 1, allée du parc Montaury, 64600 Anglet \\ didier.rihouey@univ-pau.fr, jeremy.dugor@univ-pau.fr
}

\begin{abstract}
Résumé :
La stabilité du trait côte est un enjeu majeur pour nombre de communes littorales du Golfe du Lion. Pour répondre à cette préoccupation grandissante, le projet européen INTERREG BEACHMED-e s'est donné pour objectif de tester des outils et stratégies de gestion du littoral pour le développement durable des zones côtières méditerranéennes. Dans le cadre de ce programme, une station de suivi vidéo a été installée à Valras (34) sur un littoral sableux en forte érosion depuis les années 60 où une série de 12 brise-lames ont été construits. Cet article présente l'application du suivi vidéo pour estimer l'impact de travaux de protection récents (brise-lames, butée de pied et rechargement sableux de 95,000 $\mathrm{m}^{3}$ ) ainsi que l'évolution morphodynamique d'un linéaire côtier et les incertitudes associées.
\end{abstract}

\begin{abstract}
:
Shoreline stability is an important issue along much of the Mediterranean's Coasts. European project MEDDOOC INTERREG BEACHMED-e focused on the strategic management of beach protection for the sustainable development of the Mediterranean coastal zone. In the framework of this project, a video monitoring system has been installed in Valras, Gulf of Lions (France). Important beach retreat has been observed for decades along the $3 \mathrm{~km}$ of Valras beaches and 12 breakwaters have been built until 2007. From January to May 2008, one similar protection, one submerged breakwaters and 95,000 $\mathrm{m}^{3}$ sand nourishment have been added. This paper presents the use of video monitoring technique to estimate the impact of engineering works.
\end{abstract}

Mots-clés : Vidéo quantification - Erosion - Trait de côte - Protection 


\section{$1 \quad$ Introduction}

La mise en place d'une gestion durable et pérenne de la problématique d'érosion nécessite d'appréhender toutes les échelles spatio-temporelles d'évolution morpho-sédimentaire. Depuis quelques années, l'utilisation de systèmes vidéo numériques pour quantifier ces évolutions constitue une alternative autonome et peu coûteuse (HOLMAN \& STANLEY, 2007) aux méthodes traditionnelles.

Le CETMEF, en partenariat avec l'Université de Pau et des Pays de l'Adour a installé en septembre 2007 un système de vidéo quantification numérique pour le suivi de l'érosion côtière sur la commune de Valras-Plage (34). Le projet, financé dans le cadre du programme européen MEDDOOC INTERREG BEACHMED-e, est piloté par le Conseil Général de l'Hérault.

Cet article présente l'application du suivi vidéo pour estimer l'impact des travaux de défense réalisés sur le littoral de Valras-Plage. La description du système vidéo est présentée en première partie. Les caractéristiques hydrosédimentaires du site d'étude ainsi que les travaux de défenses réalisés sont exposées dans la section 3. Les résultats d'un suivi vidéo hebdomadaire du trait de côte durant la période des travaux sont analysés dans la section 4. La précision de la méthode, ses limitations et les perspectives de développement sont ensuite discutées.

\section{Description de la technique de mesure vidéo}

\subsection{Système d'acquisition d'images}

Une multitude de processus côtiers ont une signature visuelle à la surface des océans. Récemment, plusieurs techniques de mesure vidéo ont été appliquées au suivi de différents systèmes côtiers (HOLMAN \& STANLEY, 2007).

Le Kosta System, développé par CASAGEC et AZTI Tecnalia, est un système de suivi vidéo du domaine côtier, permettant d’obtenir des données synchronisées à fréquence variable (jusqu'à $2 \mathrm{~Hz}$ ). Une station Kosta System est composée de plusieurs caméras, disposées à proximité de la côte sur un point élevé. Elle permet d'acquérir deux types d'images :

- des image instantanées : images « snap shot»,

- des images exposées sur 10 minutes, pour un intervalle d'une seconde : images «timex».

Lors de l'installation, plusieurs points de contrôle, clairement observables sur l'image brute, sont relevés sur le terrain à partir d'un DGPS centimétrique. Ces points sont ensuite utilisés pour convertir les coordonnées 2D de l'image en coordonnées 2D du monde réel à partir d'une technique de photogrammétrie (TRUCCO \& VERRI, 1998). 


\subsection{Technique de photogrammétrie}

Le principe de la vidéo quantification appliquée au suivi des environnements côtiers est basé sur une technique de photogrammétrie (TRUCCO \& VERRI, 1998) qui permet de transformer une « image oblique brute » (Figure 1.a) en une « image métrique plan » (Figure 1.b). Ce traitement préalable des images, repose sur une relation entre les coordonnées $(\mathrm{u}, \mathrm{v})$ de l’image et la position $(\mathrm{x}, \mathrm{y}, \mathrm{z})$ correspondante dans le monde réel.

a)

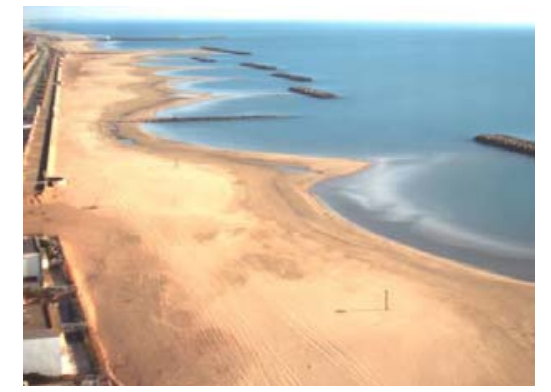

b)

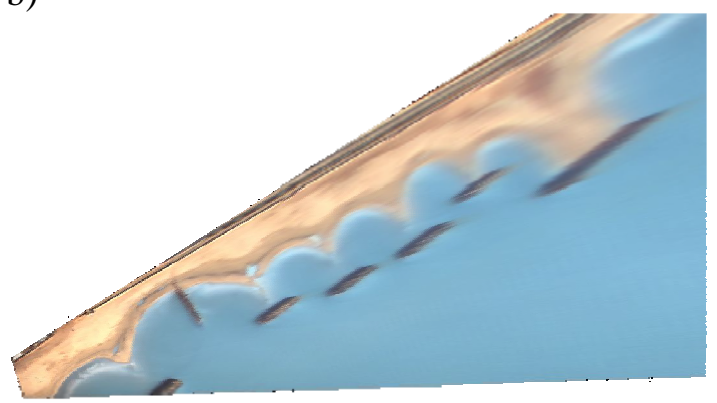

Figure 1 - Image oblique brute (a) et Image métrique plan (b) de Valras-Plage

\subsection{Détection du trait de côte}

Une fois les images sélectionnées, une Région d’Intérêt (RI) est définie manuellement (Figure 2.a). Les pixels contenus à l'intérieur de cette zone sont traités à partir d'un algorithme de détection de contour, basé sur une technique de segmentation (MORICHON et al., 2007), qui va permettre de séparer les pixels en deux groupes distincts: un groupe concernant les pixels «secs » de la partie sableuse et un groupe concernant les pixels «humides » de la partie océanique (Figure 2.b).Une nouvelle matrice binaire de la RI peut être générée grâce aux deux groupes distincts obtenus. Un algorithme de détection de contour de type SOBEL est alors appliqué à la nouvelle matrice binaire pour déterminer la position de la ligne de côte (Figure 2.c).

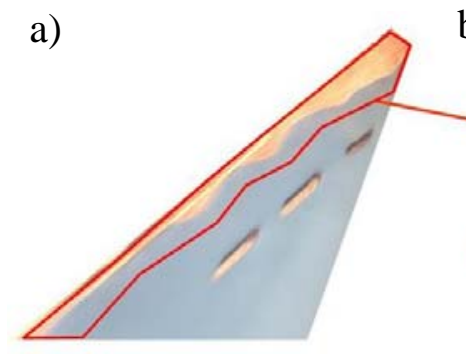

b)

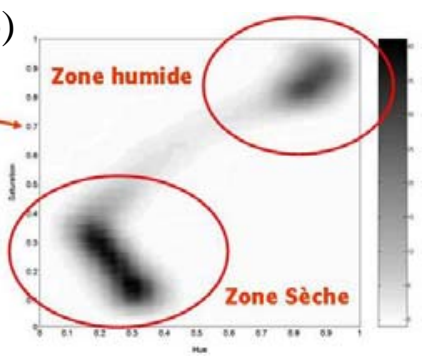

c)

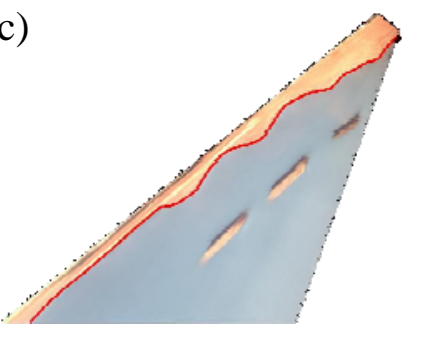

Figure 2 - (b) Analyse fréquentielle $2 \mathrm{D}$ des pixels contenus dans la Région d'Intérêt (a) et détection du trait de côte (c) 


\section{$3 \quad$ Présentation du site d'étude}

\subsection{Situation géographique et problématique}

Valras-Plage est une commune située au Sud-ouest du département héraultais sur un littoral sableux en forte érosion depuis les années 60 depuis la construction d'une digue portuaire à l'Ouest de l'embouchure de l'Orb créant un obstacle à la dérive littorale (Figure 3). La construction successive d'une série d'ouvrages longitudinaux et transversaux en aval de la dérive a réduit ponctuellement l'effet de l'érosion. Parmi les facteurs additionnels de vulnérabilité, l'urbanisation s'est déployée sur le cordon dunaire, le privant ainsi de toute protection naturelle face aux évènements océano-météorologiques de forte amplitude.
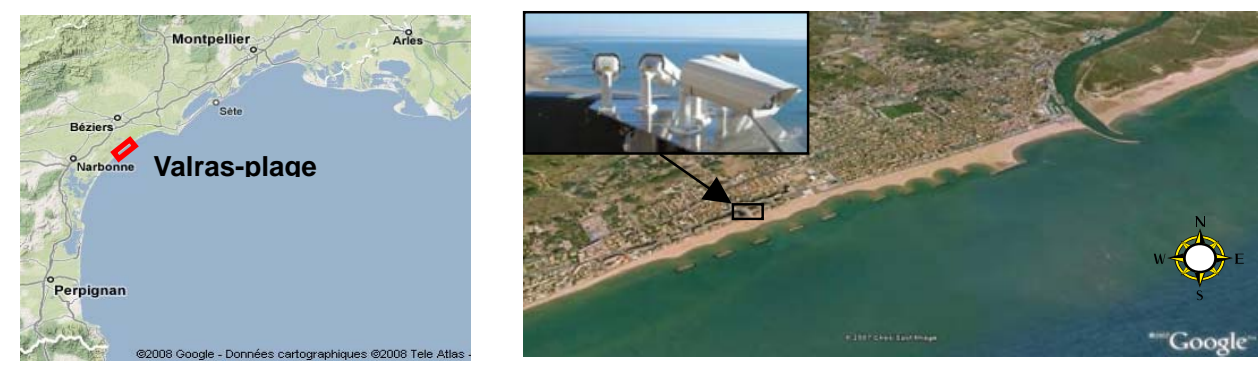

Figure 3 : Situation géographique de Valras-Plage

Cette zone fait actuellement l'objet de travaux de protection importants: construction de 3 nouveaux brise-lame (2 en 2007 cf. Figure 4.a et 1 en 2008 cf. Figure 4.b), de 2 butées de pied (Figure 4.c) et rechargement (Figure 4.b) de $95.000 \mathrm{~m}^{3}$ qui s'est achevé au printemps 2008 (Figure 4.c).

a)

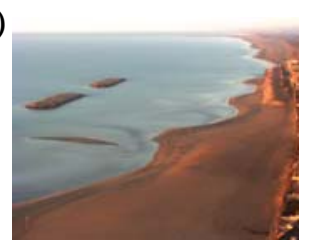

b)

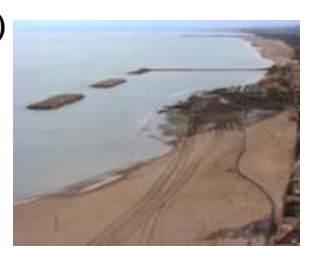

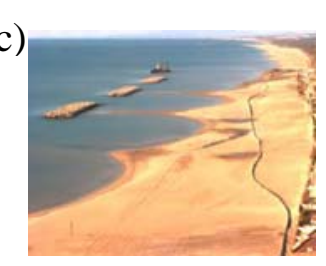

Figure 4 : Suivi des travaux de protection réalisés sur le littoral de Valras-Plage

\subsection{Conditions hydrodynamiques}

Le Golfe du Lion est caractérisé par un marnage microtidal (amplitude maximale de $0,4 \mathrm{~m}$ ) de type semi-diurne à inégalité diurne (systématiquement une des deux marées quotidiennes est moins intense que l'autre). L'agitation y est donc dominée par des états de mer de provenance sud sud-est et est nord-est dont la hauteur significative moyenne est de $0,83 \mathrm{~m}$ et la période moyenne de $5 \mathrm{~s}$ (CERTAIN, 1999). Le fetch important de cette partie de la Méditerranée permet cependant le développement de fortes houles pouvant excéder $4 \mathrm{~m}$ de hauteur significative. 


\subsection{Description des stations vidéo}

Le nombre de caméras, leur hauteur et la distance focale des lentilles utilisées conditionnent l'emprise et la précision des images vidéo. La station vidéo de Valras-Plage (Figure 4.a) est située au sommet d'une tour résidentielle, à une altitude de $47 \mathrm{~m}$. Elle est constituée de 6 caméras CCD de 0,8 Mega pixels. Le système couvre un linéaire côtier de $4 \mathrm{~km}$ (Figure 6) avec une résolution transversale sub-métrique au niveau du trait de côte (Figure 5.a) et une résolution longitudinale métrique à $300 \mathrm{~m}$ de la station et d'environ $20 \mathrm{~m}$ par pixel à $1,5 \mathrm{~km}$ de la station (Figure 5.b).

a)

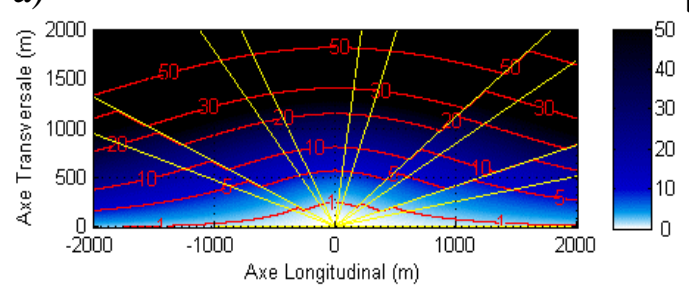

b)

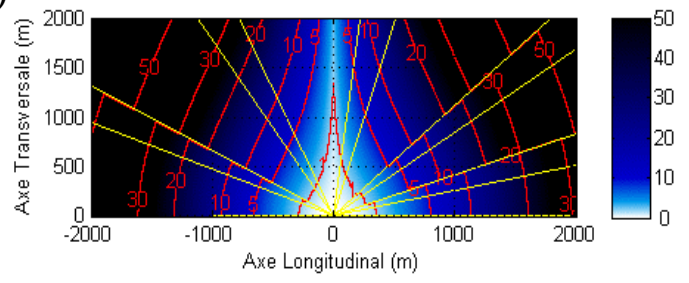

Figure 5. Carte de résolution (a) transversale et (b) longitudinale de la station vidéo Valras-Plage.

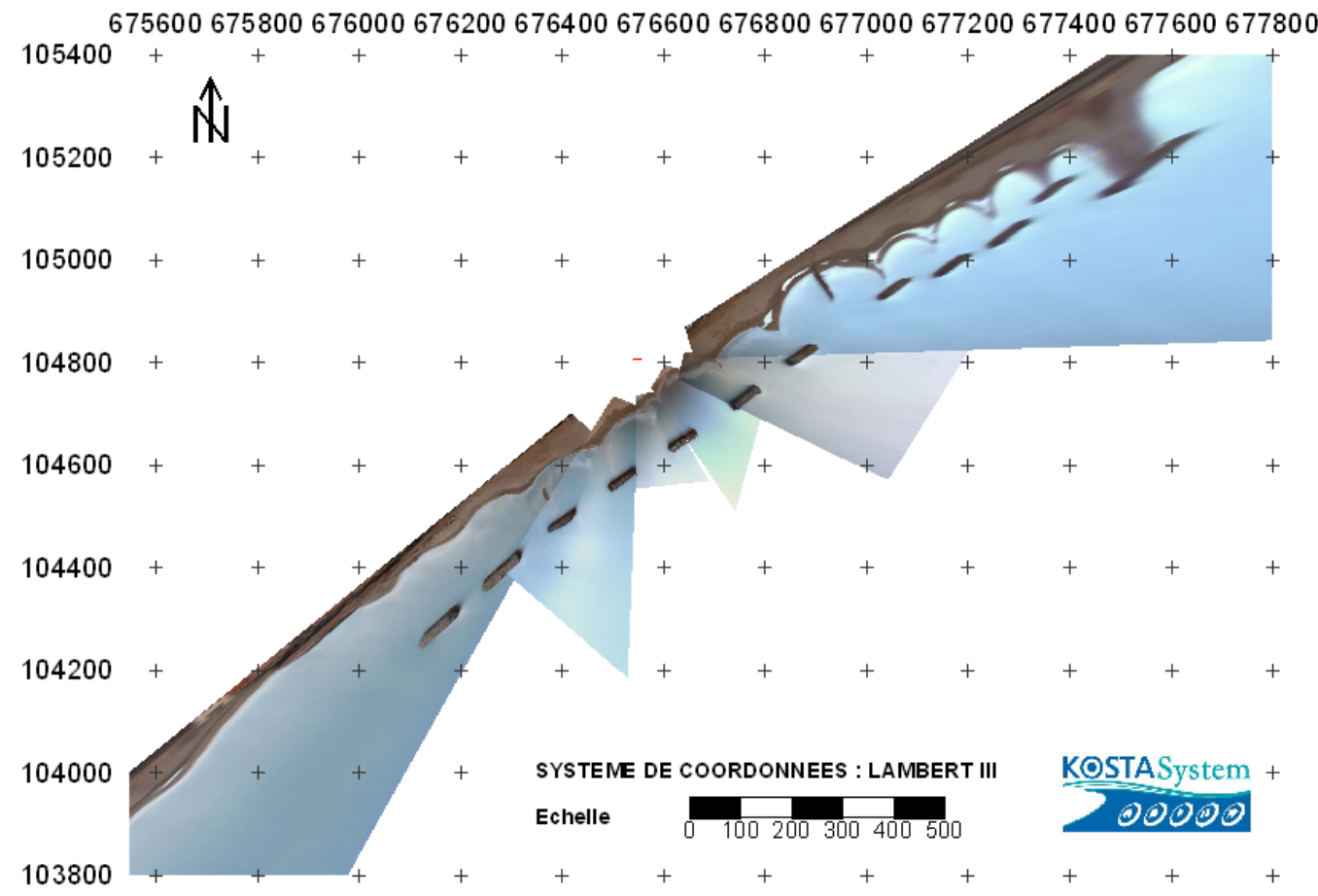

Figure 6 : Intégration SIG d'une image métrique plan panoramique issue des 6 caméras de la station vidéo de Valras-Plage 


\section{$4 \quad$ Résultats}

\subsection{Suivi du trait de côte}

Dans cette étude, le terme trait de côte, est utilisé pour définir la position instantanée de l'interface eau / terre, fonction du niveau de marée, de la pression atmosphérique, du vent et des conditions d'agitation. L'analyse des données marégraphiques est donc primordiale pour l'exploitation des images vidéo (marégraphe le proche de Valras-plage : Sète).

Afin de discriminer évolution «naturelle» et impact anthropique des travaux de protection, sont étudiées séparément la partie Est (Figure 7.a) de la partie Ouest (Figure 7.b) du littoral de Valras-Plage. De décembre 2007 à Juin 2008, 19 traits de côte (Figure 9.a et 9.b) ont été détectés par la technique de segmentation décrite en section 2.3 .
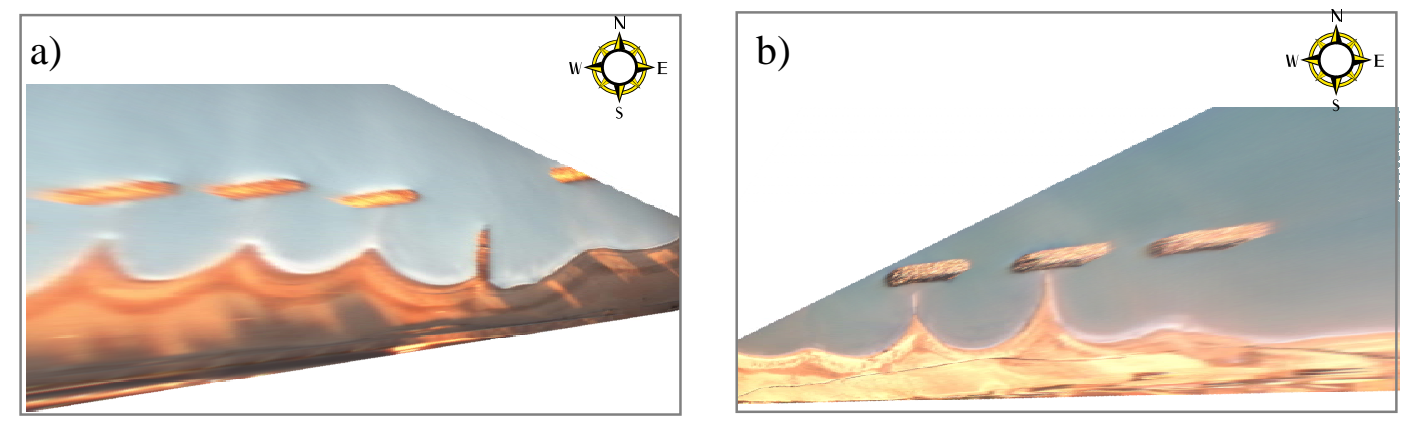

Figure 7 : Images métriques plan de a) Valras-Plage Est et b) Valras-Plage Ouest

Pour limiter l'influence du niveau d'eau sur la position des traits de côte détectés, les images utilisées ont été sélectionnées de façon à minimiser les différences relatives de niveau d'eau. La Figure 8 représente les données marégraphiques à Sète durant la période d'étude ainsi que les 19 dates (traits verticaux) utilisées pour le suivi du trait de côte. La variation entre niveaux d'eau le plus bas et le plus haut au cours de la période d'étude est de 20 cm.

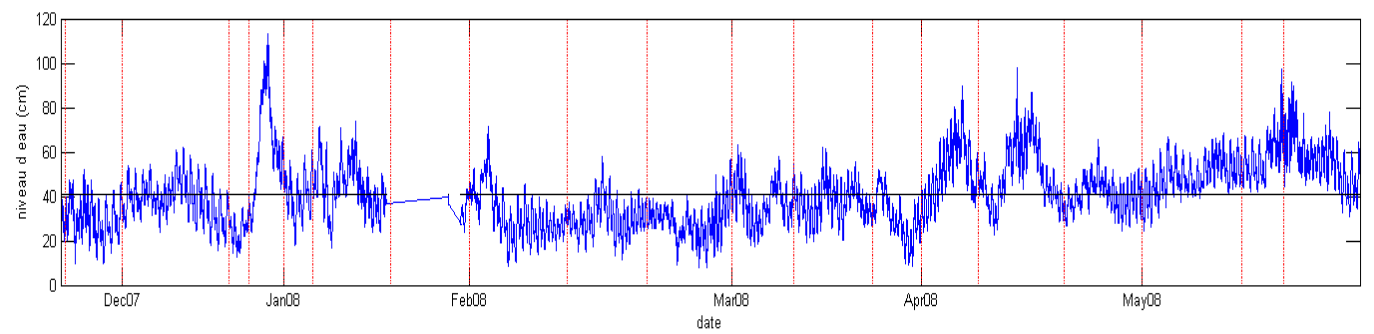

Figure 8 : Chronique du niveau d'eau relevé au marégraphe de Sète au cours de la période d'étude. Dates utilisées pour le suivi du trait de côte (traits verticaux)

(Source SHOM) 
Une analyse statistique simple met en évidence la position moyenne du trait de côte (trait rouge, cf. Figures 9.c et 9.d) et l'amplitude des évolutions du trait de côte au cours des 6 mois d'observation (positions maximales et minimales respectivement en noir et bleu, cf. Figures 9.c et 9.d). Les figures 9.e et 9.f qui représentent l'écart type des évolutions respectives de la partie Est et Ouest mettent en évidence le rechargement artificiel de sédiment effectué durant la période d'étude. Il est à noter toutefois que l'écart type intègre les évolutions du trait de côte et l'erreur induite par les variations du niveau d'eau, évalué ci-après.
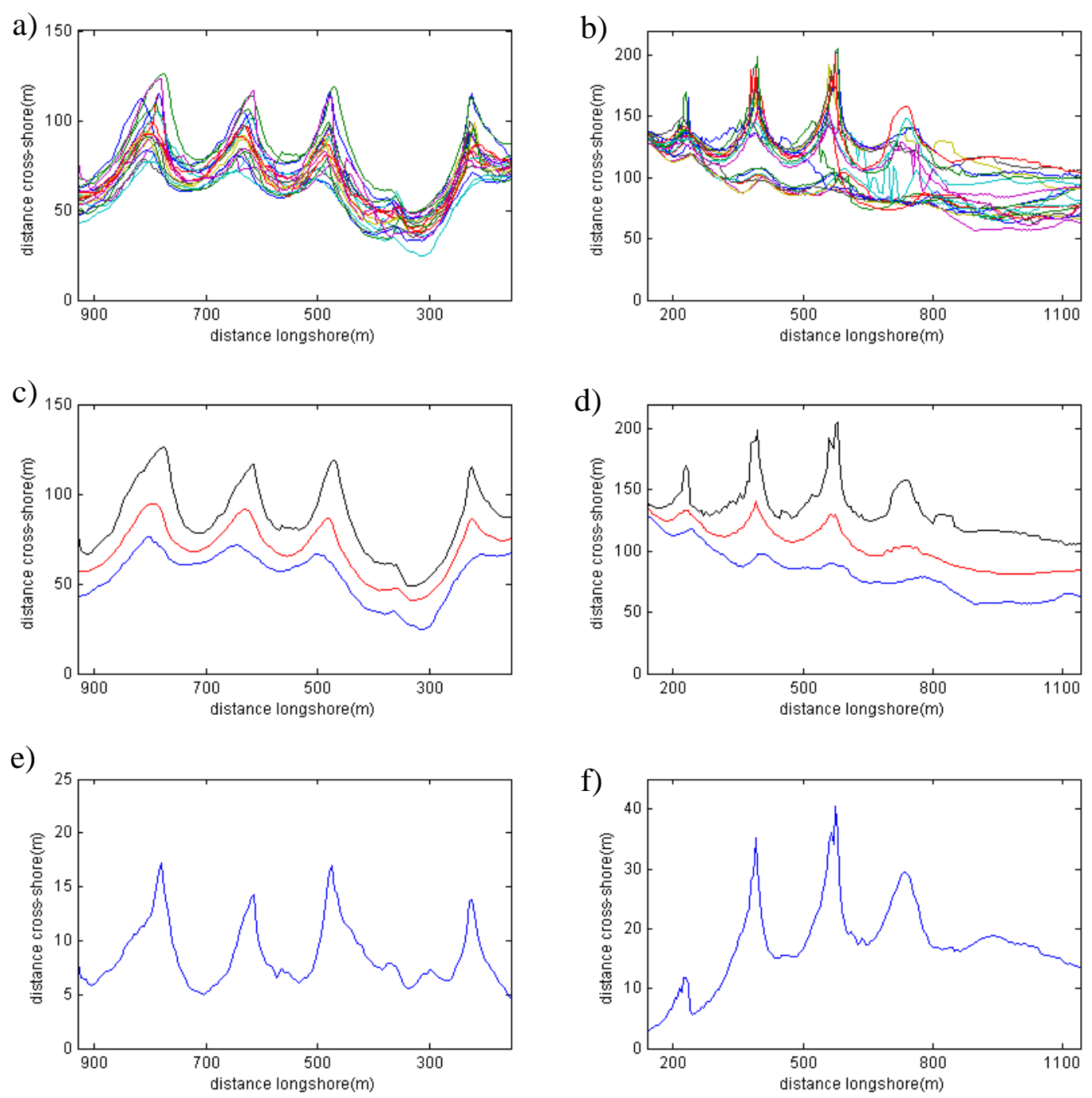

Figure 9 : Suivi du trait de côte.

Série temporelle du trait côte de a) Valras-Plage Est et b) Valras-Plage Ouest. Position maximum, moyenne et minimum du trait de côte à l'Est c) et l'Ouest d). Ecart-type de la série temporelle de traits de côte à l'Est e) et l'Ouest f). 


\subsection{Evaluation des erreurs / calcul des surfaces de plage}

Lors de la détection du trait de côte, l'erreur induite par les variations du niveau d'eau est d'autant plus importante que la pente de la plage est faible. Les pentes de plage les plus faibles se situent généralement dans l'axe des tombolos (Figure 9.a.). Pour estimer l'erreur induite par les variations du niveau d'eau deux profils de plage issus d'une bathymétrie effectuée après les travaux fin Mai 2008 (Figure 9.b.), sont comparés sur la Figure 9.b.
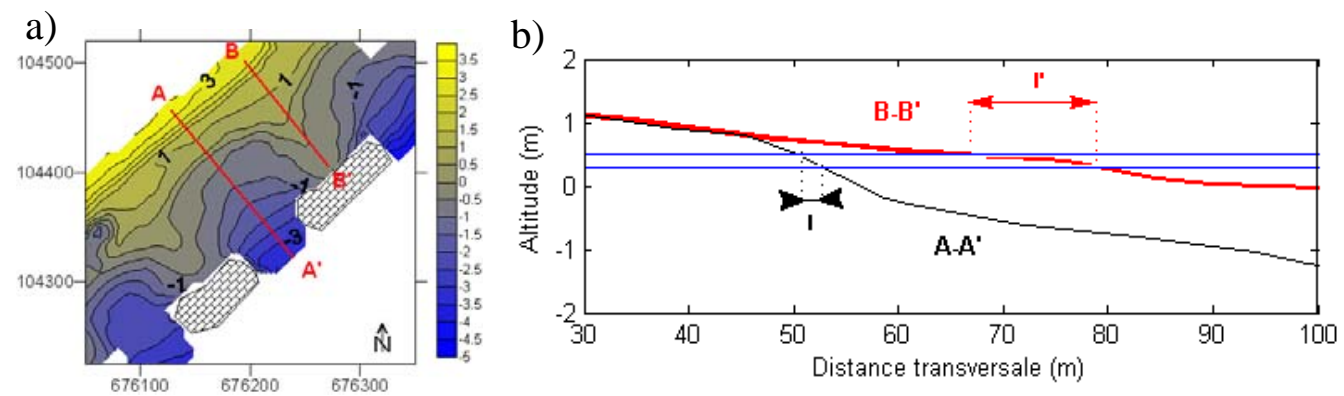

Figure 9 : Influence relative de la pente de plage et du niveau d'eau sur la position du trait de côte : a) bathymétrie et b) profils cross-shore.

La variation entre le niveau d'eau le plus bas et le plus haut au cours de la période d'étude étant de $20 \mathrm{~cm}$ (section 4.1), l'erreur transversale induite sur la position du trait de côte est de $2 \mathrm{~m}$ sur le profil A-A' et de $12 \mathrm{~m}$ sur le profil B-B' (Figure 9.b). Ce résultat corrobore l'amplitude de l'écart type des évolutions de la partie Est (Figure 8.e).

a)

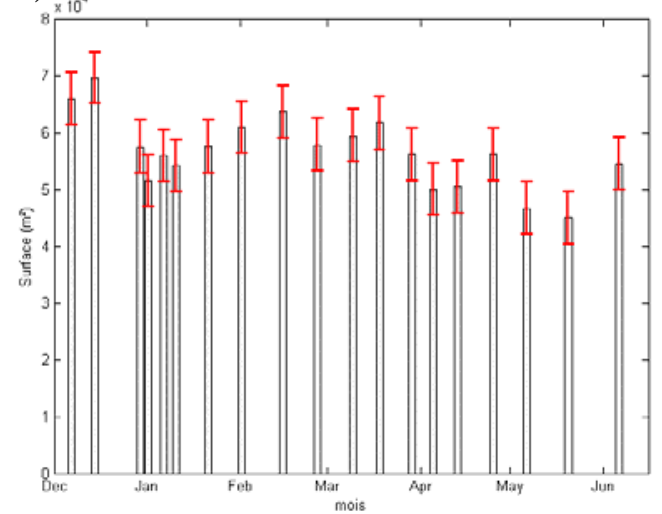

b),

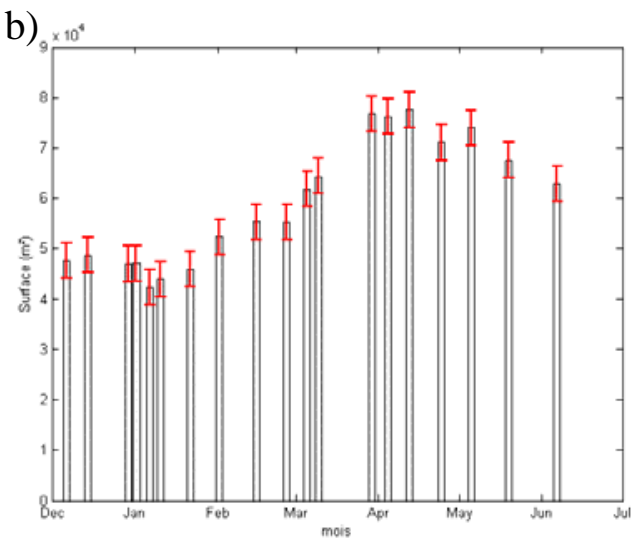

Figure 10 : Evolution de surfaces de a) Valras-Plage Est et b) Valras-Plage Ouest

Pour mettre en évidence les tendances globales d'évolution du trait de côte, les surfaces de plage (Figure 10.a et 10.b) sont calculées à partir de la série de traits de côte présentés dans la section 4.1 (Figure 9.a et 9.b). 
Pour estimer l'erreur induite par les variations du niveau d'eau sur le calcul des surfaces de la partie Est de la plage $E_{\text {Surf-Est }}$ (barre d'erreur cf. Figure 10a), on détermine alors l'écart type représenté sur la Figure 8.e.

Sous l'effet des rechargements sableux, l'écart type de la partie Ouest (Figure 8.f) présente une amplitude plus de deux fois supérieure à celle mesuré à l'Est. Ainsi, pour estimer l'erreur induite par les variations du niveau d'eau sur le calcul des surfaces de la partie Ouest de la plage on utilise la relation suivante :

$$
E_{\text {Surf-Ouest }}=E_{\text {Surf-Est }} \frac{\Delta x_{\text {Ouest }}}{\Delta x_{\text {Est }}}
$$

Où $\Delta x_{E s t}$ et $\Delta x_{\text {Ouest }}$ sont respectivement les longueurs de trait de côte étudier à l’Est et à l’Ouest.

Ces calculs d'erreurs, qui surestiment probablement l'erreur réelle, permettent de mettre en évidence l'alternance de périodes d'accrétion / érosion sur la partie Est du littoral (Figure 10.a) sous l'effet des tempêtes illustrées par la Figure 8.

Sur la partie Ouest, un gain d'environ $30.000 \mathrm{~m}^{2}$ est mesuré sous l'effet de l'apport de $95.000 \mathrm{~m}^{3}$ de sable. De plus, une redistribution du stock sédimentaire est observée après les rechargements.

\section{$5 \quad$ Conclusions}

Un système de vidéo quantification numérique pour le suivi de l'érosion côtière a été installé en Septembre 2007 sur la commune de Valras-Plage (34) où de nombreux travaux de défense ont été réalisés début 2008. Une détection hebdomadaire de la position du trait de côte a été mise en œuvre durant la durée des travaux. L'estimation de l'erreur induite par les variations du niveau d'eau sur le calcul des surfaces a permis de mettre en évidence des tendances d'évolution « naturelle » et l'impact des rechargements sableux.

La poursuite du suivi sera particulièrement intéressante pour suivre et quantifier dans le temps la redistribution des sables de rechargement, l'évolution du trait de côte dans la zone d'ombre des brises lames et également en aval dérive où le littoral, plus vulnérable, est protégé par des ouvrages immergés.

Remerciements : Mairie de Valras-Plage, Syndic Bel Horizon, Météo France (données vents), SHOM et DRELR (données marégraphiques). 


\section{$6 \quad$ Références bibliographiques}

1 CERTAIN, R. (2002). Morphodynamique d'une côte sableuse macrotidale à barres : le golfe du Lion (Languedoc-Roussillon). Thèse de doctorat, Université de Perpignan,.

2 HOLMAN R. A., STANLEY, J. (2007). The history and technical capabilities of Argus. Coastal Engineering, 54, 477-492.

3 MACQUEEN J. (1967). Some methods for classification and analysis of multivariate observations. Proceedings of 5th Berkeley Symposium on Mathematical Statistics and Probability, Berkeley, University of California Press, 1, 281-297.

4 MORICHON, D., DAILLOUX, D., AARNINKHOF, S., ABADIE, S. (2007). Using a shore based video system to hourly monitor storm water plumes (Adour River, Bay of Biscay). Journal of Coastal Research, 24, 133-140.

5 TRUCCO, E., VERRI, A. (1998). Introductory Techniques for 3-D Computer Vision. Prentice Hall, Inc. 